\title{
Est-il critique que les pharmaciens soignent les malades en phase critique?
}

\author{
par Clarence Chant
}

$\mathrm{L}$ es soins aux malades en phase critique ont vu le jour avec le travail de Florence Nightingale durant la guerre de Crimée en $1854^{1}$, mais la sous-spécialité médicale des soins aux malades en phase critique est toutefois relativement jeune. C'est en 1958 qu’apparaît aux États-Unis la première unité de soins intensifs (USI) multidisciplinaire, et ce n'est qu'en 1986 que l'American Board of Medical Subspecialties a reconnu la sous-spécialité médicale des soins aux malades en phase critique ${ }^{2}$. Les services de soins pharmaceutiques aux malades en phase critique ont vu le jour vers les années $1970^{3}$, et ont évolué durant les 40 années qui suivirent pour devenir l'une des sphères de pratique principales pour les pharmaciens cliniciens, qui a d'ailleurs sa propre section au sein de la Society of Critical Care Medicine (SCCM), la plus importante association professionnelle internationale dans le domaine ${ }^{3}$.

L'utilité des pharmaciens dans les soins des malades en phase critique est bien documentée ${ }^{4}$. Diverses études ont montré que les pharmaciens soignant les malades en phase critique contribuent à réduire les erreurs de médication, à améliorer les résultats thérapeutiques, à réduire les dépenses et le gaspillage, et à diminuer les taux de mortalité chez les patients souffrant de maladies thromboemboliques ou d'infections ${ }^{4-6}$. Les pharmaciens soignant les malades en phase critique sont aussi reconnus comme essentiels par les acteurs en dehors de la profession de pharmacien, comme la SCCM. En fait, plusieurs lignes directrices de la SCCM décrivent le champ de pratique et le rôle des pharmaciens parmi les meilleurs modèles de pratique pour la composition du personnel des USI ${ }^{7,8}$. L'un des leaders du mouvement pour la sécurité des patients, le docteur Peter Pronovost, a affirmé que l'intégration des pharmaciens à l'équipe soignante des malades en phase critique est l'une des six caractéristiques de l'USI permettant d'améliorer les résultats cliniques?.

Deux articles dans le présent numéro du JCPH viennent bonifier le nombre croissant des publications qui démontrent les divers rôles des pharmaciens dans les soins des malades en phase critique. Dans une enquête sur la fréquence et les types d'erreurs associées à l'administration de médicaments intraveineux dans les USI, Summa-Sorgini et ses collaborateurs ${ }^{10}$ ont mis en lumière un taux d'erreur de 14,3\% ou 3,0 erreurs par perfusion. Cette étude souligne le rôle potentiel des pharmaciens dans les soins des malades en phase critique quant à l'amélioration de la qualité, à la détection des erreurs et à la production de travaux d'érudition. Walker et ses collaborateurs ${ }^{11}$ décrivent en détail la mise sur pied d'une équipe de gestion responsable des antimicrobiens dans une USI et la manière dont le travail de l'équipe vient compléter les efforts actuels des pharmaciens dans la surveillance des traitements antimicrobiens des patients de l'USI.

Il semblerait donc que les pharmaciens soient un besoin fondamental pour toute USI, parce qu'ils prennent part à divers aspects des soins et des activités d'érudition liés aux patients en phase critique, qu'ils sont bien acceptés par leurs pairs et que leur travail est associé à l'amélioration des résultats cliniques, économiques et humanistes ${ }^{4}$. En revanche, dans un sondage mené en 2006 aux États-Unis auquel ont répondu 382 hôpitaux totalisant 1034 USI, seulement 62,2\% des répondants ont déclaré avoir des pharmaciens (à temps plein ou partiel) assignés exclusivement à une USI ${ }^{12}$ et participant aux tournées de soins auprès des patients pour une moyenne de 4,4 jours par semaine. Moins de la moitié (41\%) des hôpitaux ont déclaré que les pharmaciens donnaient des conseils thérapeutiques aux médecins en dehors des heures de service de jour habituelles et seulement 18,2\% des répondants offraient les services d'une pharmacie satellite 24 heures par jour. Ces résultats corroborent ceux d'une enquête internationale menée par LeBlanc et ses collaborateurs ${ }^{13}$ qui ont signalé que, ailleurs qu'aux États-Unis, $74,4 \%$ des 134 pharmaciens soignant les malades en phase critique participaient aux tournées médicales.

Alors, pourquoi un service dont les bienfaits sont démontrés est-il absent de si nombreux hôpitaux? Et dans le cas des hôpitaux qui ont des pharmaciens assignés exclusivement aux soins des malades en phase critique, pourquoi ces pharmaciens ne sont-ils présents qu'une partie de la journée, alors que 
d'autres membres de l'équipe de l'USI sont présents en tout temps? La prestation des soins aux malades en phase critique par les pharmaciens n'est-elle " critique " qu'à seulement certains moments? Le concept de la prestation continue des services pharmaceutiques aux malades en phase critique n'est certes pas nouveau ${ }^{14}$. Plusieurs facteurs expliquent les raisons pour lesquelles les pharmaciens ne sont pas présents en tout temps, notamment un manque de ressources financières ou de personnel adéquatement formé, l'inégalité de la consignation des activités des pharmaciens dans les dossiers médicaux, l'absence d'un modèle de pratique commun et peut-être aussi (quoique contre-intuitif) l'opinion des administrateurs à la fois des hôpitaux et des services de pharmacie que les soins des pharmaciens aux malades en phase critique ne sont pas réellement essentiels ou qu'ils sont simplement une extension du produit (c.-à-d., les médicaments), plutôt que d'être étroitement associés au patient. Cela va à l'encontre de l'avis d'un groupe de travail de la SCCM, chargé de définir le meilleur modèle de pratique pour une USI, qui a classé les données probantes pour ce qui est des avantages procurés par les soins des pharmaciens aux malades en phase critique (niveau C) en tête de celles concernant les autres professionnels de la santés. Tout comme les « lacunes dans les soins » des patients qui ne reçoivent pas les traitements fondés sur des données probantes qui leur permettraient d'améliorer leur état de santé, on ne réussira à combler cette « lacune de pharmaciens » que par des efforts concertés non seulement de la profession, mais aussi d'autres parties prenantes. Bien que certains facteurs, comme le manque de ressources financières, puissent être en dehors du contrôle des pharmaciens, d'autres facteurs, notamment la consignation plus systématique des activités du pharmacien dans les dossiers médicaux et plus de travaux d'érudition démontrant les contributions des pharmaciens (dont ceux apparaissant dans ce numéro du Journal), peuvent et doivent être gérés de façon plus constante par tous les pharmaciens soignant les malades en phase critique. Ce n'est que par ces efforts que nous réaliserons la vision du professeur Joseph Dasta, l'un des pionniers des soins pharmaceutiques aux malades en phase critique, qui a décrit l'avenir des pharmaciens soignant les malades en phase critique en ces termes : « les rôles et les responsabilités du pharmacien seront élargis pour ainsi assurer l'exactitude et la pertinence de l'information clinique, économique et pharmacogénétique sur les médicaments contenue dans des systèmes d'information intégrés... [et] l'expression 'pharmacien clinicien' sera remplacée par 'gestionnaire des connaissances pharmacothérapeutiques de l'USI'. Le pharmacien sera remboursé pour la prestation de ces services d'une façon similaire à celle des médecins et du personnel infirmier. ${ }^{3}$ Voyons à ce que la prestation des soins aux malades en phase critique par les pharmaciens soit « critique » en tout temps!

[Traduction par l'éditeur]

\section{Références}

1. Grenvik A, Pinsky MR. Evolution of the intensive care unit as a clinical center and critical care medicine as a discipline. Crit Care Clin 2009;25(1): 239-250.

2. History of critical care. Mount Prospect (IL): Society of Critical Care Medicine; 2001-2011 Publié au : www.sccm.org/AboutSCCM/History_ of_Critical_Care/Pages/default.aspx. Consulté le 3 novembre 2011.

3. Dasta J. Critical care. Ann Pharmacother 2006;40(4):736-737.

4. Kane SL, Weber RJ, Dasta JF. The impact of critical care pharmacists on enhancing patient outcomes. Intensive Care Med 2003;29(5):691-698.

5. MacLaren R, Bond CA. Effects of pharmacist participation in intensive care units on clinical and economic outcomes of critically ill patients with thromboembolic or infarction-related events. Pharmacotherapy 2009; 29(7):761-768.

6. MacLaren R, Bond CA, Martin SJ, Fike D. Clinical and economic outcomes of involving pharmacists in the direct care of critically ill patients with infections. Crit Care Med 2008;36(12):3184-3189.

7. Rudis MI, Brandl KM; Society of Critical Care Medicine and American College of Clinical Pharmacy Task Force on Critical Care Pharmacy Services. Position paper on critical care pharmacy services. Crit Care Med 2000;28(11):3746-3750.

8. Brilli RJ, Spevetz A, Branson RD, Campbell GM, Cohen H, Dasta JF, et al.; American College of Critical Care Medicine Task Force on Models of Critical Care Delivery. Critical care delivery in the intensive care unit: defining clinical roles and the best practice model. Crit Care Med 2001;29(10):2007-2019.

9. Randolph AG, Pronovost P. Reorganizing the delivery of intensive care could improve efficiency and save lives. J Eval Clin Pract 2002;8:1-8.

10. Summa-Sorgini C, Fernandes V, Lubchansky S, Mehta S, Hallett D, Bailie T, et al. Errors associated with IV infusions in critical care. Can J Hosp Pharm 2012;65(1):19-26.

11. Elligsen M, Walker SAN, Simar A, Daneman N. Prospective audit and feedback of antimicrobial stewardship in critical care: program implementation, experience, and challenges. Can J Hosp Pharm 2012; 65(1):31-36

12. Maclaren R, Devlin JW, Martin SJ, Dasta JF, Rudis MI, Bond CA. Critical care pharmacy services in United States hospitals. Ann Pharmacother 2006;40(4):612-618.

13. LeBlanc JM, Seoane-Vazquez EC, Arbo TC, Dasta JF. International critical care hospital pharmacist activities. Intensive Care Med 2008; 34(3):538-542.

14. Hall K, Guay M. The implementation of comprehensive critical care pharmacy services through the development of a 24-hour satellite pharmacy. Can J Hosp Pharm 1982;35(6):184-188.

Clarence Chant, Pharm. D., BCPS, FCSHP, FCCP, est spécialiste et leader en pharmacie clinique au St Michael's Hospital, à Toronto, en Ontario. Il est également rédacteur adjoint du JCPH.

Adresse de correspondance :

Dr Clarence Chant

Pharmacy

St Michael's Hospital

30 Bond Street, Room Q4036

Toronto ON M5B 1W8

\section{Courriel : CHANTC@smh.ca}

\section{Remerciements}

L'auteur désire remercier le professeur Joseph Dasta qui a révisé cet article avant qu'il ne soit soumis pour publication. 